\title{
A comparative clinical trial to evaluate the effect of mouthwash containing "BIO Sp. White" system
}

\author{
Molochkov Anton $V^{1}$, Kondyurova Evgenia $V^{2}$, Eliseykina Elena $V^{2}$, Vilikotskiy Anatoliy $E^{3 *}$, Yagafarova Irina $E^{3}$ and Zakirova Svetlana $A^{3}$ \\ ${ }^{1}$ Moscow Scientific and Practical Center of Dermatology, Venereology and Cosmetology of Moscow City Health Department, 2nd Spinning St., Moscow, 105037, \\ Russia
}

${ }^{2}$ N. P. Ogarev's Mordovia State University, Bolshevik st., 68, Saransk, 430005, Rep. Mordovia

${ }^{3}$ SPLAT GLOBAL LLC, Lotte Plaza, Novinskiy Boulevard, 8, Moscow, 121099, Russia

\begin{abstract}
Objective: The aim of this study was to evaluate and compare efficacy of "SPLAT Professional Sensitive" mouthwash with and without BIO Sp.White Mouthwash system.

Methods: This open one-center comparative study was carried out during $28 \pm 3$ days on 40 volunteers. All volunteers were divided into 2 groups in a 1: 1 ratio. The volunteers at visit 1 carried out brushing their teeth for 2 minutes, then the rinse was used for 30 seconds, and the hygiene index was measured before and after. Then, the volunteers used the products 2 times a day for 2 minutes, in the morning and in the evening, for 4 weeks with an intermediate visit to the dentist on the 14th day of the study. The comparison was made with the initial values of the parameters. All volunteers received a Splat Professional Complete toothbrush of medium hardness. Participants were forbidden to use other means of oral hygiene (toothbrushes, toothpastes, rinses).

Results: It was shown that after 4 weeks of using product with and without BIO Sp.White Mouthwash system there was a decrease in inflammation and gums bleeding (PMA and SBI indices) for both groups by $64.17 \%$ and $75.19 \% ; 63.9 \%$ and $62.1 \%$, respectively. The four-week product use led to a significant decrease the tooth sensitivity (54.53\% and 61.28\%) and OHI-S index (68.97\% and 76.19\%) in group 1 and 2, respectively. Whitening effect was more pronounced after use of the product with BIO Sp.White Mouthwash system (11.46\% vs 5.67\%). Compared to baseline, there was the considerable strengthening of enamel according to TER results $(52.78 \%$ and $59.14 \%)$. In addition, a significant deodorizing effect were noted. The oral microbiota analysis showed that the number and species diversity of pathogenic microorganisms decreased in both groups.
\end{abstract}

Conclusion: The results suggest that due to the original composition, the "SPLAT Professional Sensitive" BIO Sp.White ${ }^{\circledR}$ Mouthwash system is able to provide soft whitening, as well as to improve the gums and the teeth condition. In the course of the study, none of the undesirable side effects were recorded.

\section{Introduction}

Demand for improving the aesthetics of the dentition, including whitening, has increased dramatically over the past two decades. Lifestyle and dieting (drinking coffee, tea, red wine and fruit juices), as well as using tobacco products, can lead to staining of teeth.

Professional cleaning and polishing of teeth or the use of whitening products for oral hygiene can help remove many types of surface stains, primarily under the influence of abrasive/polishing agents. For deeper color changes, peroxide bleaching techniques are used. These methods are based on the use of hydrogen peroxide applied directly to the tooth surface, or produced by a chemical reaction of carbamide peroxide or sodium perborate [1]. In spite of peroxide efficacy, adverse effects have become evident. Cervical root resorption and tooth sensitivity are possible consequence of internal bleaching and are more frequently observed in teeth treated with the thermo-catalytic procedure [2]. In fact, tooth sensitivity is experienced in $15-78 \%$ of patients undergoing tooth bleaching [3].

The use of additional chemical agents that can enhance tooth cleaning can improve whitening effect in products for oral care. Many substances, including enzymes, have been previously studied for their stain removal efficiency. Since external stains are mainly incorporated into the oral biofilm, it is possible that enzymes, such as proteases, can help break down colored films and potentiate their removal [4]. It has been established that bromelain from Ananas comosus is a safe and effective proteolytic enzyme and has an anti-adhesive effect on oral microorganisms [5]. In addition, unlike other proteolytic enzymes, for example, papain, bromelain does not cause the destruction of immunoglobulin molecules, thus does not reduce the activity of the immune system [6].

While dental plaque forming, a pronounced change in the composition of the microflora occurs, which can cause such diseases as caries, gingivitis and periodontitis.

${ }^{*}$ Correspondence to: Vilikotskiy Anatoliy E, SPLAT GLOBAL, LLC, Novinskiy Boulevard, 8121099, Moscow, Russia, E-mail: vilikotskiy@splat.ru

Key words: mouthwash, oral care, single-center comparative study, bromelain, bifidobacterium lysate

Received: November 08, 2019; Accepted: November 18, 2019; Published: November 21, 2019 
Diseases of the oral cavity are reflected not only in the cosmetic effect; they significantly affect the quality of life, the social aspects of human life, and determine the development of some systemic pathologies. As a rule, smoking, an abnormal diet, or poor oral hygiene lead to such conditions. Mouth rinses help control biofilm bacterial balance [7].

One of the most effective ways to prevent dental plaque is the use of lysates of microorganisms that make up the biofilm [8]. The antigens in lysates stimulate the activity of the factors of immune-biological resistance of the organism: particles of probiotic bacteria give a biochemical signal to the normal microflora of the oral cavity, ensuring the survival of the latter and the intensification of their reproduction. Use of bacterial lysates allows to normalize the inflammatory status of the tissues of the oral cavity and to restore the ratio of microorganisms indicated for a healthy oral cavity. Moreover, using lysates, instead of live bacteria, has several advantages, namely high survival rate during production and storage that eliminates the risk of the formation of an acid that creates a cariogenic environment in the oral cavity. An additional advantage is that, unlike living probiotics, lysates are unable to tolerate antibiotic resistance genes, and therefore do not contribute to the spread of antibiotic resistance. Purposeful inclusion of bacterial lysate in the complex for the oral care allows for effective prevention of both gingivitis, periodontitis, caries, as well as bacterial, fungal and viral infections of the oral mucosa, tongue, upper respiratory tract, halitosis, glossitis, post-traumatic inflammation caused by orthodontic structures and other diseases involving the immune system and the microbiota of the oral cavity and upper respiratory tract.

The role of antigens in the BIO Sp.White mouthwash system is played by lysates of the Bifidobacterium spp., Lactobacillus spp. and Streptococcus spp. The mechanism of lysates anti-inflammatory action on mucous membranes is associated with the expansion of the population of regulatory T-lymphocytes (Treg), which is accompanied by the suppression of the synthesis of pro-inflammatory cytokines TNF $\alpha$ and MCP-1, IL-12 and IFN $\gamma$, and an increase in the "antiinflammatory" cytokine IL-10. Membrane structures of lysates are recognized by TLR2 and TLR9 receptors of immune cells, and Nod receptors, resulting in increased expression of cyclooxygenase COX-2 and prostaglandin PGE-2 and reducing the level of oral mucosa cells apoptosis $[9,10]$.

The synergy of bacterial lysates and bromelain in BIO Sp.White complex is believed to lead to a powerful enhancement of the cleansing action, as well as the effects on the microbial cavity and the restoration of homeostasis of the immune system, ensuring the prevention of diseases of the oral cavity.

With this background, this study aimed at compare the effectiveness of two mouthwash formulations, one of which contains bifidobacterium lysate and bromelain (BIO Sp.White Mouthwash system), and the second one has the same formulation without these ingredients.

\section{Materials and methods}

\section{Test formulations}

"SPLAT Professional Sensitive" BIO Sp.White Mouthwash system (Mouthwash A) contained bromelain and bifidobacterium lysate and "SPLAT Professional Sensitive" without BIO Sp.White Mouthwash system (Mouthwash B-Placebo) not containing abovementioned components were manufactured by Organic Pharmaceuticals (Novgorod region, Russia).

\section{Study design}

Open one-center comparative study.

The duration of the study was $28 \pm 3$ days. The study included 40 volunteers. All patients received a medium hardness Splat Professional Complete toothbrush. All volunteers were divided into 2 groups in a 1: 1 ratio. The first group volunteers used Mouthwash $\mathrm{A}$, and the second one used Mouthwash B.

The volunteers at visit 1 carried out brushing their teeth for 2 minutes, then the rinse was used for 30 seconds, and the hygiene index was measured before and after. Next, patients used the products 2 times a day for 2 minutes, in the morning and in the evening, for 4 weeks (visit 3) with an intermediate visit to the dentist on the 14th day of the study (visit 2).

Participants were forbidden to use other means of oral hygiene (toothbrushes, toothpastes, rinses).

\section{Study population}

After a screening session, patients were selected on the basis of the following criteria:

1. Volunteers aged 18 to 50 years.

2. The number of teeth is at least 20 .

3. The presence of tooth sensitivity (index of the intensity of teeth hyperesthesia, IIGZ $\geq 2$ ).

Exclusion criteria were:

1. Professional teeth cleaning and whitening less than 90 days before the examination.

2. Carrying out other dental procedures, including orthodontic and surgical, less than 30 days prior to the study, or the need to carry out them during the study period.

3. The use of antimicrobial agents less than 90 days prior to inclusion.

4. The use oflocal antiseptics intended for the mouth or throat, including part of the rinse and toothpastes (for example, chlorhexidine). The exceptions are toothpastes and rinses containing plant extracts or essential oils.

5. Continuous use of anti-inflammatory drugs, toothpastes and rinses containing plant drugs, toothpastes and rinses containing plant extracts or essential oils.

6. Conditions and diseases that may impede the participation of the patient in the study or may affect the assessment of the studied parameters.

7. Hepatitis B or C, HIV infection.

8. Chemotherapy, radiation therapy or cytokine therapy less than 5 years before being included in the study; Hypersensitivity to the components of the investigated products or food allergies.

9. Any somatic diseases that may affect the mineralization of enamel.

10. Pregnancy or lactating.

\section{Clinical evaluation}

\section{OHI-S index}

To evaluate the cleaning effect of studied products Oral Hygiene Index Simplified (OHI-S) (Greene and Vermilion, 1960) was used. The 
index was measured on visit 1 before and after 2 minutes of cleaning, on visits 2 and 3 . To determine the index, 6 teeth were examined: 16, 11, 26, 31-vestibular surfaces 36, 46-lingual surfaces. Evaluation of dental plaque was carried out visually or with the help of staining solutions (Schiller-Pisarev, Fuchsin, Erythrosine). Determination of supra- and subgingival calculus was carried out using a dental probe. The calculation of the index consisted of the values obtained for each component of the index, divided by the number of examined areas by summing up both values.

\section{Index of the intensity of teeth hyperesthesia}

For objective assessment of the sensitivity of hard tooth tissue, an index of the intensity of teeth hyperesthesia (IIGZ) was used (Shtorina GB, 1986). The index was calculated by the formula 1 :

IIGZ = The sum of the index values of each tooth / Number of teeth with increased sensitivity (1)

The index is calculated in points, which are determined based on the following indicators:

0 - no response to temperature, chemical and tactile stimuli;

1 point - the presence of sensitivity to temperature stimuli;

2 points - the presence of sensitivity to temperature and chemical stimuli;

3 points - the presence of sensitivity to temperature, chemical and tactile stimuli.

\section{A visual analogue scale}

A visual analogue scale is proposed for self-assessment by the patient of the level of pain in the samples, where the values from 1 to 3 corresponded to the light intensity of pain, from 4 to 6 points moderate, and 7 or higher - strong.

\section{Tooth color and whitening effect analysis}

Evaluation of tooth color and whitening effect of the studied products was carried out by comparing the results obtained by the Toothguide 3D-Master scale on visits 1 and 3. Before and after applying the products, 6 upper front teeth were evaluated according to the Toothguide 3D-Master scale.

\section{Test of the enamel resistance}

The enamel remineralization effect was carried out by means of test of enamel resistance (TER). The vestibular surface of one of the upper central incisors was cleaned of plaque using a cotton ball moistened with hydrogen peroxide solution, and then the tooth was dried with a cotton ball. The tooth was isolated from the saliva with cotton rolls. A drop of $1 \% \mathrm{HCl}$ solution with a diameter of $1.5-2 \mathrm{~mm}$ was applied to the center of the vestibular tooth surface with a micropipette. After 5 seconds, the drop was washed with distilled water. The tooth surface was dried with a cotton ball. On the area of enamel, which was treated, 1 drop of $1 \%$ solution of methylene blue was put. The dye was immediately removed with a dry cotton ball in one motion, tightly pressing the ball to the surface of the tooth.

In daylight, the color of the treated area was compared to the shades of a standard 10-point scale. Estimated acid resistance of enamel in points from 1 to 10 (from pale blue to dark blue). The procedure was carried out on visits 1 and 3 .
According to the result, the patient was referred to one of 4 dispensary groups:
1-3 points - high acid resistance of enamel;
4-5 points - moderate acid resistance of enamel;
6-7 points - low acid resistance of enamel;
more than 7 points - very low acid resistance of enamel.

\section{Anticaries effect}

The anti-caries effect was identified by the difference in the results of measuring the DMF index on visits 1 and 3. DMF index is the sum of all surfaces of teeth on which caries or filling was diagnosed in one individual. (If a tooth is removed, then in this index it is considered to be 5 surfaces). In determining the index, the early forms of dental caries in the form of white and pigmented stains were not take into account.

\section{PMA index}

To evaluate anti-inflammatory effect the PMA index proposed by Schour and Massler (1947) was used. PMA is measured up to a 2-minute cleansing, after 2 and 4 weeks of application of the test product. Cotton swab dipped in dye Pisarev-Schiller, lubricate the surface of the gums. If there is inflammation, the gums acquire a certain color. Further, the index was evaluated.

\section{SBI index}

To evaluate the hemostatic effect of studied products Sulcus Bleeding Index (SBI) (Muhlemann and Son, 1971) was determined. The SBI is measured up to 2 minutes of cleansing and after 2 and 4 weeks of application of the test product.

\section{Deodorizing effect}

To evaluate the deodorizing effect an unscaled 10-centimeter visual analogue scale was used.

Measurements were carried out on a visit 1 before and after applying the product, as well as after 14 and 28 days of use. The left edge corresponded to 0 , the right edge corresponded to 10 . The researcher assessed the smell from the patient's mouth at a distance of $20 \mathrm{~cm}$ and put a mark on the scale. Next, by applying the ruler to the scale, estimating the number of centimeters from the left edge and rounding to integer values, then a score of the intensity of breath from the mouth is estimated.

\section{The saliva collection}

The saliva collection for ELISA and microbiota analysis was carried out in both groups of volunteers before cleaning on visit 1 and on visit 3 to sterile Eppendorf tubes and then the tubes were frozen.

\section{ELISA}

Standard ELISA protocol was used to measure the level of immunoglobulins IgM, IgG, IgA (total), IgA (secretory).

\section{Microbiological study}

The microbiota analysis was carried out using microscopic and bacteriological methods. Sampling of fluid (saliva) was carried out using probe swabs for sampling, transporting and storing biological samples complete with Ames test medium. Sowing was carried out on Petri dishes with Colombian agar with the addition of defibrinated blood and incubated in a thermostat at $37^{\circ} \mathrm{C}$ for $20-24$ hours under 
conditions of $15 \% \mathrm{CO}_{2}$ content. The identification of microorganisms was carried out according to the Order of the Ministry of Health of the USSR No. 535 dated 04.22.1985.

\section{Safety assessment}

Safety assessment was performed taking into account the frequency and severity of adverse reactions.

\section{Ethical aspects}

This study was carried out in accordance with the Helsinki Declaration of the World Medical Association "Ethical principles of medical research involving human subjects as a subject of research" from 1964 with subsequent amendments and guidelines of the International Conference on Harmonization - Good Clinical Practice (ICH GCP).

\section{Results}

Table 1 and 2 shows the average clinical indices for two groups. A decrease in the amount of plaque and tartar, a decrease in tooth sensitivity and gum inflammation, as well as an increase the acid resistance of tooth enamel were observed, during the 4 weeks of "SPLAT Professional Sensitive" with and without BIO Sp.White Mouthwash system using (Figure 1). In addition, there were strong deodorizing and whitening effects for both mouthwash formulations (Figure 2).

The OHI-S index for two groups dropped to $21.6 \%$ and $22.7 \%$ for visit 1, to $45.74 \%$ and $49.13 \%$ for visit 2, to $68.97 \%$ and $76.19 \%$ for visit

Table 1. The average clinical indices for group 1 (with the BIO Sp.White ${ }^{\circledR}$ Mouthwash system)

\begin{tabular}{|c|c|c|c|c|}
\hline \multicolumn{5}{|c|}{ Group 1 (with the BIO Sp.White ${ }^{\circledR}$ Mouthwash system) } \\
\hline Index name & Baseline & Visit 1 & Visit 2 & Visit 3 \\
\hline OHI-S & $2.76 \pm 0.49$ & $2.16 \pm 0.36^{*}$ & $1.50 \pm 0.38^{*}$ & $0.86 \pm 0.46^{*}$ \\
\hline IIGZ & $2.54 \pm 0.34$ & $2.16 \pm 0.36^{*}$ & $1.61 \pm 0.41^{*}$ & $1.1 \pm 1.16^{*}$ \\
\hline VAS & $7.05 \pm 1.87$ & $5.26 \pm 1.66^{*}$ & $3.68 \pm 1.16^{*}$ & $2.00 \pm 1.25^{*}$ \\
\hline PMA & $37.20 \pm 19.47$ & - & $21.56 \pm 13.49^{*}$ & $13.33 \pm 7.51^{*}$ \\
\hline SBI & $3.60 \pm 0.94$ & & $2.15 \pm 0.59^{*}$ & $1.30 \pm 0.66^{*}$ \\
\hline TER & $5.40 \pm 1.19$ & - & - & $2.55 \pm 1.28^{*}$ \\
\hline $\begin{array}{c}\text { Deodorizing } \\
\text { effect }\end{array}$ & $6.85 \pm 1.52$ & $5.25 \pm 1.52^{*}$ & $3.45 \pm 1.28^{*}$ & $2.05 \pm 0.94^{*}$ \\
\hline Whitening effect & $1.92 \pm 0.72$ & & & $1.70 \pm 0.73^{* *}$ \\
\hline Anticaries effect & $14.40 \pm 3.47$ & - & - & $14.40 \pm 3.47$ \\
\hline
\end{tabular}

OHI-S $=$ Oral Hygiene Index Simplified, IIGZ $=$ Hyperesthesia Intensity Index (Shtorina index), VAS = visual analogue scale, PMA = Papillary Marginal Alveolar Index, SBI = Sulcus Bleeding Index, TER $=$ Test of the Enamel Resistance

${ }^{*} \mathrm{p}<0.01, * * \mathrm{p}<0.05$ compared to baseline

Table 2. The average clinical indices for group 2 (without the BIO Sp.White ${ }^{\circledR}$ Mouthwash system)

\begin{tabular}{|c|c|c|c|c|}
\hline \multicolumn{5}{|c|}{ Group 2 (without the BIO Sp.White ${ }^{\circledR}$ Mouthwash system) } \\
\hline Index name & Baseline & Visit 1 & Visit 2 & Visit 3 \\
\hline OHI-S & $2.31 \pm 0.33$ & $1.79 \pm 0.44$ & $1.18 \pm 0.5$ & $0.55 \pm 0.37$ \\
\hline IIGZ & $2.26 \pm 0.41$ & $1.87 \pm 0.47 *$ & $1.00 \pm 0.47 *$ & $0.88 \pm 0.65^{*}$ \\
\hline VAS & $6.00 \pm 1.00$ & $4.58 \pm 0.77^{*}$ & $2.90 \pm 0.79^{*}$ & $1.70 \pm 0.66^{*}$ \\
\hline PMA & $39.83 \pm 6.65$ & - & $24.09 \pm 4.14^{*}$ & $9.88 \pm 4.34^{*}$ \\
\hline SBI & $3.30 \pm 1.13$ & & $1.95 \pm 0.76^{*}$ & $1.25 \pm 0.44^{*}$ \\
\hline TER & $4.65 \pm 1.39$ & - & - & $1.90 \pm 0.85^{*}$ \\
\hline $\begin{array}{c}\text { Deodorizing } \\
\text { effect }\end{array}$ & $6.85 \pm 1.52$ & $5.25 \pm 1.52 *$ & $3.45 \pm 1.28^{*}$ & $2.05 \pm 0.94^{*}$ \\
\hline Whitening effect & $2.12 \pm 0.69$ & & & $2.00 \pm 0.62 * *$ \\
\hline Anticaries effect & $13.45 \pm 3.47$ & - & - & $13.45 \pm 3.47$ \\
\hline
\end{tabular}

OHI-S $=$ Oral Hygiene Index Simplified, IIGZ $=$ Hyperesthesia Intensity Index (Shtorina index), VAS = visual analogue scale, PMA = Papillary Marginal Alveolar Index, SBI = Sulcus Bleeding Index, TER $=$ Test of the Enamel Resistance

${ }^{*} \mathrm{p}<0.01, * * \mathrm{p}<0.05$ compared to baseline

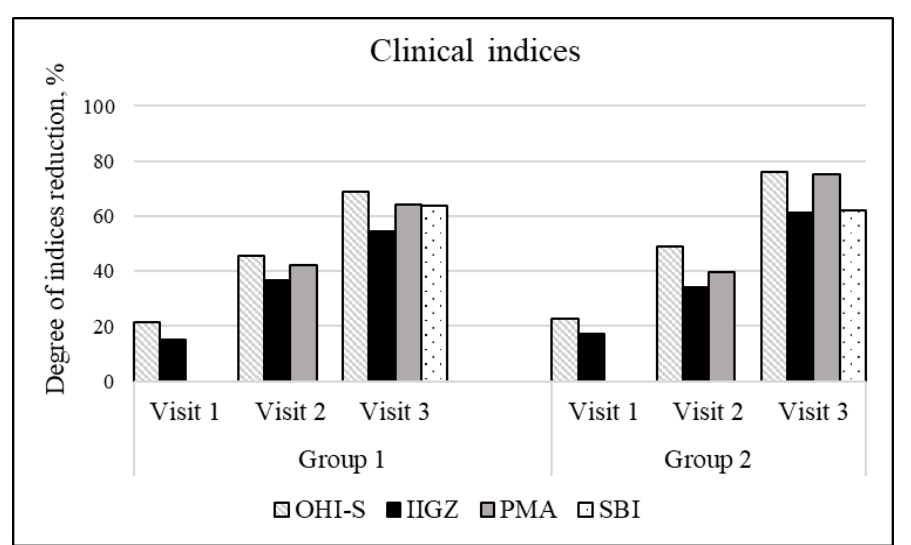

Figure 1. Degree of the hygienic, gingival and periodontal indices reduction

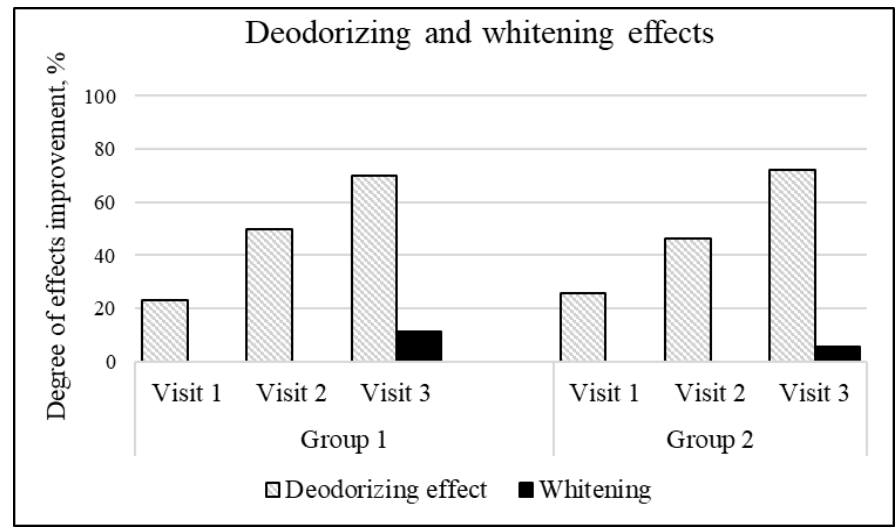

Figure 2. Degree of the deodorizing and whitening effects improvement

3 , respectively. Tooth sensitivity parameters significantly improved, Shtorina index in both groups decreasing by $15.16 \%$ and $17.26 \%$ for visit 1 , by $54.53 \%$ and $61.28 \%$ for visit 3 , respectively. The results of the visual analogue scale also showed improvement in desensitizing effect after the first use of both mouthwash formulations, and by the visit 3 it reached $71.64 \%$ and $71.56 \%$ for groups 1 and 2 , respectively. The condition of the gums improved significantly by day 28 of clinical investigation, as indicated by a decrease in the PMA index by $64.17 \%$ and $75.19 \%$ and in the SBI index by $63.9 \%$ and $62.1 \%$ in two groups, respectively. Fourweek use of the product led to a significant strengthening of enamel, which is reflected in the increase of the teeth acid resistance in the TER test by $52.78 \%$ in the first and by $59.14 \%$ in the second group. Furthermore, there was a significant improvement in oral odor in both groups throughout the experiment. After the first visit the deodorizing effect reached $23.36 \%$ and $25.89 \%$ for two groups, and by the third visit, odor improvement amounted $70.07 \%$ and $72.32 \%$, respectively. The powerful refreshing effect was confirmed by the survey results, as $80 \%$ of volunteers determined the degree of deodorizing effect above the average with a moderate or long duration. The oral microbiota analysis showed that in both groups the number and species diversity of pathogenic microorganisms significantly decreased after two weeks of product application. The data described did not have statistical differences between the two experimental groups.

The results of the whitening effect study showed that in group 1 the color of the enamel was lightened by $11.5 \%$, which is 2 times better than in group 2. An examination of the oral cavity immune status (Table 3) demonstrated that the use of the BIO Sp.White Mouthwash system statistically significantly reduced the secretory immunoglobulin A 
Table 3. The average of the oral cavity immune status markers for two groups

\begin{tabular}{|c|c|c|c|c|}
\hline & \multicolumn{2}{|c|}{ Group 1 } & \multicolumn{2}{c|}{ Group 2 } \\
\hline Index name & Baseline & Visit 3 & Baseline & Visit 3 \\
\hline IgM & $0.234 \pm 0.413$ & $0.215 \pm 0.246$ & $0.161 \pm 0.139$ & $0.215 \pm 0.246$ \\
\hline IgG & $0.026 \pm 0.026$ & $0.015 \pm 0.011$ & $0.020 \pm 0.023$ & $0.018 \pm 0.017$ \\
\hline IgA (total) & $0.043 \pm 0.025$ & $0.041 \pm 0.028$ & $1.005 \pm 0.298$ & $0.898 \pm 0.388$ \\
\hline IgA (secretory) & $1.022 \pm 0.373$ & $0.760 \pm 0.368 * *$ & $0.041 \pm 0.025$ & $0.031 \pm 0.016$ \\
\hline
\end{tabular}

$* * \mathrm{p}<0.05$ compared to baseline.

expression by $25 \%$, what could be the result of the anti-inflammatory effect. No side effects were observed throughout the study.

\section{Discussion}

The present clinical study was undertaken to evaluate and compare the effects of the "SPLAT Professional Sensitive" Mouthwash system and without "SPLAT Professional Sensitive" BIO Sp.White Mouthwash system. Modern rinses should have a number of characteristics, such as the ability to reduce tooth sensitivity, good refreshment, as well as gentle bleaching without side effects. The study examined the cleansing, anti-inflammatory, anti-caries, deodorizing, whitening and desensitizing actions, as well as the effect on the immunity and oral microbiota, of the "SPLAT Professional Sensitive" Mouthwash system and without "SPLAT Professional Sensitive" BIO Sp.White" Mouthwash system. Results showed these products to significantly improves oral hygiene and the color of tooth enamel, as well as has a beneficial effect on the gums and decreases the enamel sensitivity. Firstly, it is worth noting that both rinses showed a powerful cleansing effect, a strong deodorizing effect, and reduced gum inflammation, as well as decreased tooth sensitivity. Further, the reduction of pathogenic microflora was also observed when using the mouthwashes. However, the effectiveness of bleaching with these two products was different; the BIO Sp.White Mouthwash system showed a 2 times more whitening effect, than a mouthwash without this system.

Thus, due to the original composition, the "SPLAT Professional Sensitive" BIO Sp.White Mouthwash system is able to provide soft whitening, as well as to improve the gums and the teeth condition.

\section{Funding}

Financial support for the conduct of the study and for editorial assistance in the preparation of the article was provided by SPLAT GLOBAL LLC.

\section{Author contributions}

All the authors contributed to the design, conduct and reporting of the study. All authors had access to the final study report, made contributions to the development of the manuscript, had final responsibility for the decision to submit, and approved the submitted version.

\section{Competing interests}

All the authors received funding from SPLAT GLOBAL LLC at the time the study was conducted.

\section{References}

1. Hillman JD, McDonell E, Hillman CH, Handfield M (2016) Dental whitening effect of an oral probiotic. Dent Oral Craniofac Res 2: 202-205.

2. Broon NJ, García SA, Zamacona DKB (2014) Intrinsic tooth whitening using thermocatalytic technique. Clinical case report 18: 186-190.

3. Tredwin CJ, Naik S, Lewis NJ, Scully C (2006) Hydrogen peroxide tooth-whitening (bleaching) products: review of adverse effects and safety issues. Br Dent $J$ 200: 371376. [Crossref]

4. Chakravarthy PK, Acharya S (2012) Efficacy of extrinsic stain removal by novel dentifrice containing papain and bromelain extracts. J Young Pharm 4: 245-249. [Crossref]

5. Patil PA, Ankola AV, Hebbal MI, Patil AC (2015) Comparison of effectiveness of abrasive and enzymatic action of whitening toothpastes in removal of extrinsic stains-a clinical trial. Int J Dent Hyg 13: 25-29. [Crossref]

6. Matharu Z, Bandodkar AJ, Guptac V, Malhotra BD (2012) Fundamentals and application of ordered molecular assemblies to affinity biosensing. Chemical Society Reviews 41: 1363-1402.

7. Oliveira JBS, Sarlo RS, Bresciani E, Caneppele T (2017) Whitening Efficacy of Whitening Mouth Rinses Used Alone or in Conjunction with Carbamide Peroxide Home Whitening. Oper Dent 42: 319-326. [Crossref]

8. Marsh PD, Head DA, Devine DA (2015) Ecological approaches to oral biofilms control without killing. Caries Res 49: 46-54. [Crossref]

9. Kashtanova DA, Egshatyan LV, Tkachyova ON (2015) The Involvement of Human Gut Microbiota in Chronic Systemic Inflammation. Clinical Microbiology and Antimicrobial Chemotherapy 17: 310-317.

10. Kolosova NG (2016) Efficiency of bacterial lysates in prevention of respiratory infections in children. Lechashchiy vrach 9: 47-47.

Copyright: (2019 Molochkov Anton V. This is an open-access article distributed under the terms of the Creative Commons Attribution License, which permits unrestricted use, distribution, and reproduction in any medium, provided the original author and source are credited. 